\title{
Biometric Template Update: An Experimental Investigation on the Relationship between Update Errors and Performance Degradation in Face Verification
}

\author{
Gian Luca Marcialis, Ajita Rattani, and Fabio Roli \\ University of Cagliari - Department of Electrical and Electronic Engineering \\ Piazza d'Armi - 09123 Cagliari (Italy) \\ \{marcialis,ajita.rattani,roli\}@diee.unica.it
}

\begin{abstract}
Current methods for automatic template update are aimed at capturing large intra-class variations of input data and at the same time restricting the probability of impostor's introduction in client's galleries. These automatic methods avoid the costs of supervised update methods, which are due to repeated enrollment sessions and manual assignment of identity labels. Most of state-of-the-art template update approaches add input patterns to the claimed identity's gallery on the basis of their matching score with the existing templates, which must be above a very high "updating" threshold. However, regardless of the value of such updating threshold, update errors do exist and impact strongly on the effectiveness of update procedures. The introduction of impostors into the galleries may degrade the performance quickly. This effect has not been studied in the literature so far. Therefore, a first experimental investigation is the goal of this paper, with a case study on a face verification system.
\end{abstract}

Keywords: Biometrics, Template update, Intra-class variations, Face biometrics, template update errors, impostors, threshold selection.

\section{Introduction}

The performance of a typical biometric system depends on the "representativeness" of the templates collected during the "enrollment" phase. Concisely,"representativeness" means the capability of enrolled template to recognize new input biometric data. In general, enrolled templates are captured in controlled environments, while input biometric data exhibit "substantial" intra-class variations. These may be (a) temporary variations like for face, changes in environment, expression, pose etc., or (b) permanent variations like for face, aging, accidental cuts or scars on subject's face, changing the subject's appearance.

To increase the templates representativeness, one can enroll multiple instances of each identity representing possible variations in input data, and repeating the process of enrollment over time [1]. However, this approach is based on supervised learning as the human supervisor assigns "identity" labels manually to the data used for updating, making the process very expensive, time consuming and inefficient [1]. Moreover, supervisor must be able to estimate possible intra-class variations in input data. 
Limitations of the supervised methods are addressed by introducing automatic template update methods based on semi-supervised learning [2-5]. These methods use both the initial set of enrolled and labelled templates and a set of unlabelled data acquired during the normal system's operation, for updating. The concept is that those unlabelled data classified (pseudo-labelled) as highly confident genuine users, i.e., genuine users with the matching score exceeding a given updating threshold, are used for updating the clients' galleries. The majority of these state-of-the-art "semisupervised methods" [2-4] can be termed as "self update" methods, as they incrementally update themselves using their own knowledge.

It is worth noting that the threshold value selected for updating plays a vital role in the performance of these template update process. As only those input data, whose matching score is above the selected threshold, are added to the claimed identity's gallery. Thus, the efficiency of method and the security level of threshold selection has a direct bearing on the performance of the template update algorithms. The operation at very high or stringent threshold, may result in a very limited capability to capture input samples characterising significant intra-class variations [5]; on the other hand, relaxing the updating threshold may cause much introduction of impostors into the template gallery thus weakening the system's performance and security [5].

Although Ref. [5] pointed out the issue of threshold selection and impostor introduction, to the best of our knowledge, no other works in the literature investigated to which extent the updating threshold selection impacts on the performance degradation, and how relevant is the introduction of impostors into the template galleries. Most of the proposed methods accept the input data at a fixed nonuser specific high threshold, corresponding to a certain working point in the ROC curve of the system. Threshold is estimated by using initial enrolled templates [2-3] and is not re-estimated after iterations of updating process. In this paper, we evaluate the performance of self update at different threshold setting methods, i.e., fixed nonuser specific threshold ("same for each user") and user-specific threshold for constant and threshold re-estimated after each iteration of updation process at different security levels: zeroFAR, $1 \% \mathrm{FAR}$ and 5\%FAR. In particular, its effect on the verification performance degradation and introduction of impostors into the client's galleries has been investigated. We also draw attention on the vulnerability of self update methods to impostors even at very stringent threshold due to presence of "difficult" clients which can be easily confused with others, and clients, whose appearance intrinsically "imitates" others. These subjects have been named "lambs" and "wolves", according to the "Doddington's zoo" [6], respectively. In particular, these so called "wolves" can be classified as genuine users even at very stringent threshold settings and can intrude into galleries of another identity, in particular, the ones of lambs, hence gradually making the template self update counter productive.

In order to advance the state of art related to automatic template update procedures and to study the behaviour of the biometric template update methods from the stated viewpoint, a case study has been done on face authentication system based on EBGM [7]. Equinox Face Database [8] is used for experimental evaluation. The paper is organized as follows: Section 2, describes the self update systems in detail. Section 3, discusses the experiments and results. Conclusions are drawn in Section 4. 


\section{The Template "Self Update" Algorithm}

In the "online" template self update algorithms [2-3], a matcher operating in monomodal environment adapts itself to the variations of the input data available during the normal system's operation. The aim of these methods is to capture the temporal and temporary intra-class variations of the input data by modifying the templates and hence enhancing the generalization performance on the novel unseen data. The procedure to update the galleries is usually one of the two commonly used techniques, a) by adding the novel sample as another instance in the gallery set of the respective client ("instance based") [4] or b) by fusing the sample with the template to form a "super template", thus embedding the information into a single template [2-3] ("prototype based"). References [4-5] perform off-line updating: input data are collected during system's operations, but the updating process is performed "offline" that is, when the batch has been collected over time and system is not operating [4-5].

The general "online" self update method can be summarized as follows:

\section{Initialization:}

Each user is enrolled with its templates to build the initial gallery and the initial system parameter ("updating threshold" $(t h)$ ) is set. The details about enrollment, feature extraction and matching processes of face verification can be found in [7].

\section{Updating Process:}

Repeat for each input data arrival

a) Compare the input data with the template(s) of the claimed identity's gallery to compute the matching score $(M S)$.

b) If $M S>=t h$

Update the template set of the respective client by either fusing with the current template or adding the input data as a separate instance into the gallery set of claimed identity

else

Reject the data

end

Specifically, the self update's internal operation can be represented by using a threshold graph $(\theta$-graph) drawn on enrolled templates and input data. The graph gradually expands on each input sample arrival by connecting the template to the sample if the matching score between them is above the threshold $\theta$. Thus, the self update graph is initialized with vertices representing enrolled templates and it gradually grows on each input data arrival by connecting the highly classified input data to current templates. The edge between two nodes represents that matching score among them is above $\theta$. Fig. 1 shows an example of $\theta$-graph build by the self updating process. Edges between vertices represent the matching score obtained, by comparing input data to current template set which is always above the acceptance threshold. The updating threshold is assumed to be 75 in this case (score normalized in $[0,100]$ interval). The initial template (left side) is connected to an input due to the score which is above the threshold (see the edge label, namely, 85). This input is added to the gallery. A novel arrival is connected to such input, and/or connected to the initial template (current templates on the basis of score value), and so on. 


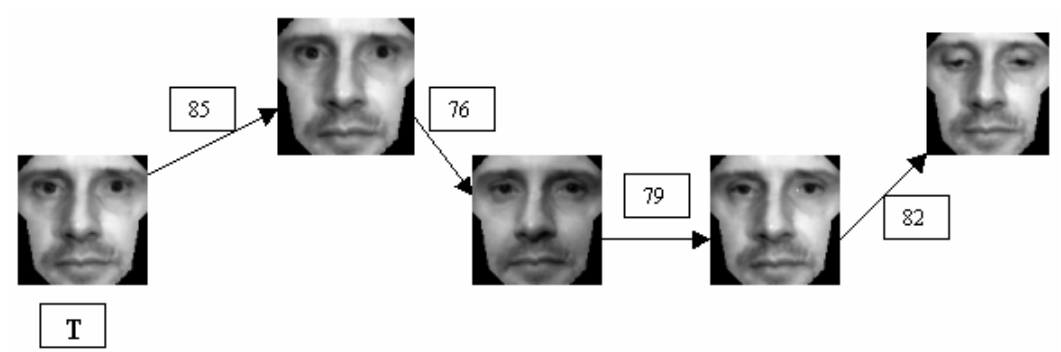

Fig. 1. The hypothetical theta graph $(\theta)$ maintained by self update's internal operation which keeps growing on the arrival of input data. The image marked $\mathbf{T}$ represents the enrolled template. The graph expands progressively by connecting the current templates to the input data, if the matching score is above acceptance threshold, which is assumed $\mathbf{7 5}$ in this example. All the input data connected to the initial template $\mathrm{T}$ (directly or through other input sample) are added to the template gallery for updating. Note that a new input data node can be linked to more than one node in the current template set on the basis of similarity above $(\theta)$. The present case only shows the connection of the input node to most similar node in template set.

Thus the connection between initial enrolled templates to highly confident examples and then the enrolled template together with the already classified examples are used to connect other samples whose scores are above $\theta$, hence the name " $\theta$ graph". Worth noting, if the same input data sequence is observed but the order is different, the corresponding $\theta$-graph is also different.

Worth noting, the out-degree of $\theta$-graph varies with $\theta$, which corresponds to the updating threshold of self update. The more is $\theta$, the less is the out-degree. Note that out-degree relates to number of images in the template gallery set which matches input sample at specific threshold. Obviously, at low threshold more images in template set will match input sample and vice-versa. Since highly genuine users are introduced in the clients' galleries for most of the template update procedures [2-5], operation at very stringent threshold, say zeroFAR, may be successful in avoiding impostors to certain extend due to very low out-degree of resulting $\theta$-graph. However, this assumption completely neglects the typical characteristics of certain clients. Doddington et al. [6] introduced some peculiar terms to indicate clients wrongly classifiable even at high thresholds $(\theta)$, namely, "lambs", and clients which can "imitate" other clients, namely, wolves. Therefore, it is reasonable to argue that the existence of wolves and lambs among clients can have an adverse impact on the self update procedure even at stringent threshold condition. In particular, a wolf into a lamb's gallery may attract other wolves, so compromising seriously the representativeness of the obtained gallery. This effect may be accentuated by the low out-degree of $\theta$-graph. These issues have not yet been studied in the literature. For many state-of-the-art methods, a fixed non-user specific threshold is set; although the access by impostors is assumed, their creeping in the templates galleries and the effect of impostors introduction has not been stated at all [1-3]. The threshold value fixed by using the initial enrolled is not re-estimated after updating iterations. To focus attention on these drawbacks, several experiments are conducted in this paper. 


\section{Experimental Results}

\subsection{Data Set and Experimental Protocol}

The data set for experimental evaluation consists of 56 individuals with 91 frontal face images representing significant intra-class variations per person, from Equinox Corporation database [8]. The face verification system and the similarity measure used are based on Elastic Bunch Graph Based Matching Algorithm (EBGM) [7].

In a typical personal verification system, a different input data owing to different access attempt, is available for each client. In order to follow this evidence, the following protocol has been adopted: one image is selected as initial template for each client. In other words, the initial gallery of each subject is made up of only one template. Threshold values are always evaluated on template set by comparing each template to the templates of all the other clients thus estimating the impostor distribution and selecting threshold at desired FAR value, being the only set available in real environments where very less labelled data are present to set the system parameters.

Remaining client images i.e., ninety, are subdivided in two sets made up of thirty (input unlabelled set of genuine users) and sixty images per client (test set of genuine users). Both unlabelled and test sets are then randomly partitioned into 56 sets, such that the $c$-th partition does not contain images of $c$-th client. Each of these partitions, consisting of thirty and sixty images, represents the "impostor set" for $c$-th client: thirty images are added to the unlabelled set and sixty to the test set. The unlabelled set contains the images which are assumed to be the input data and each image in the unlabelled set is passed to the system for verification and accordingly on the basis of matching score between input image and the templates, the self update is performed. The test set evaluates the actual improvement in generalization of the system reached by self update techniques. Equal probability of genuine and impostors attempts are assumed by keeping equal number of genuine and impostors in unlabelled and test sets. Each experiment is repeated five times for different order of sequence of input data arrival (from "unlabelled set") to minimize the effect of order of sequence of input data arrival and related results are averaged.

The experiments are carried out using the above database characteristics and experimental protocol. In order to better study the impact of threshold selection on the capture of impostors or the difficult pattern representing intra-class variations, updating process in the current experiments is carried out by adding the input data as the separate instance in the gallery set of claimed identity. During updating, the input sample score is evaluated as the maximum value among scores computed between the sample and each template into the current gallery.

\subsection{Results}

The goal of our experiments is to evaluate the reliance of impostors introduction on various threshold selection conditions like fixed non-user specific vs. user specific. Each selection type is evaluated at different updating thresholds: very stringent; zeroFAR, stringent; $1 \%$ FAR, and relaxed; 5\% FAR. Threshold can also be updated at each iteration, or kept fixed after the initial estimation on the available galleries. Thus, 
to show differences in the performance improvement and the progressive presence of impostors into the gallery by varying the initial experimental settings of template update, four threshold settings reported in Table 1 are investigated.

Table 1. Threshold settings adopted in reported experiments

\begin{tabular}{|c|c|}
\hline \multicolumn{2}{|c|}{ Threshold Settings } \\
\hline Along the algorithm iterations & Along clients \\
\hline Fixed & Non user specific \\
\hline Updated & User specific \\
\hline
\end{tabular}

For each experiment, two curves are reported and compared: Equal Error Rate (EER) on the test set at each updating iteration; \% impostors into the galleries at each iteration. This is also an approximated average evaluation of the $\theta$-graph out-degree. These curves are drawn for each threshold setting in combination (Table 1) in a plot, at three security levels: zeroFAR, $1 \%$ FAR, $5 \%$ FAR.

Figs. 2(a-f) show these plots. In particular:

1. Figs. 2(a-b). At zeroFAR updating threshold, there is no appreciable performance enhancement of face verification system for all the threshold settings methods (Table 1). Benefits of user-specific threshold appeared at the late iterations, as more amount of samples have been exploited in comparison to other threshold settings. The minimum EER value can be clearly noticed as a function of certain number of samples exploited for updating. With the increase in the number of samples exploited after certain iterations, EER starts increasing. By comparing Fig. 2(a) and Fig. 2(b), some correspondences can be found with the percentage impostors into the gallery, whose trend is always increasing, and the performance degradation depicted in Fig. 2(a). It can be hypothesised that, till the decreasing trend is noticed in EER value of Fig. 2(a), the genuine users are added into the gallery, thus the effect of impostors is not evident. This effect becomes prominent after the gradual accumulation of impostors, due, probably, to the cumulative "attraction" of impostors from other ones previously introduced ("wolves"?) in the same galleries. From the performance degradation viewpoint, it is worth noting that the difference in the impostor percentage of $1 \%$ (see values from 100 to 150 exploited samples in Fig. 2(b)) caused a remarkable decrease of EER (Fig. 2(a)).

2. Figs. 2(c-d). Observations similar to the previous ones can be drawn here also regarding impostors introduction. The minimum value of EER is lower than that of Fig. 2(a). This is due to the number of exploited samples, as can be seen by comparing Figs. 2(b, d). Moreover, the differences among threshold settings are more remarkable. Adopting an user-specific updated threshold appeared to be the best strategy in this case because it has apparently allowed to reduce the number of impostors introduced into the galleries. However, the trend of impostors introduction in Fig. 2(d) has increased very fast: from $4 \%$ impostors introduced in Fig. 2(b) to $20 \%$ impostors introduced there.

3. Figs. 2(e-f). For all threshold settings, the downward trend in performance is noticed even at the initial stages of updating. The worst performance is obtained by adopting a fixed and non user specific threshold, but, from the viewpoint of 
impostors introduced, substantial differences with other settings cannot be appreciated. The amount of impostors introduction exceeds 50\%, thus making self update very counter productive in this case. For both points 2 and 3, the $\theta$-graph analysis is in our opinion superfluous, since the related out-degree is large even at the initial iterations of the algorithm, and impostors can be easily attracted.

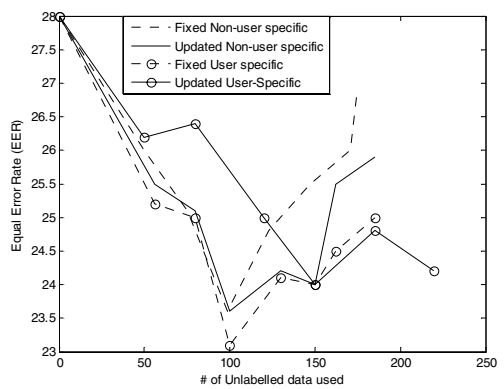

(a)

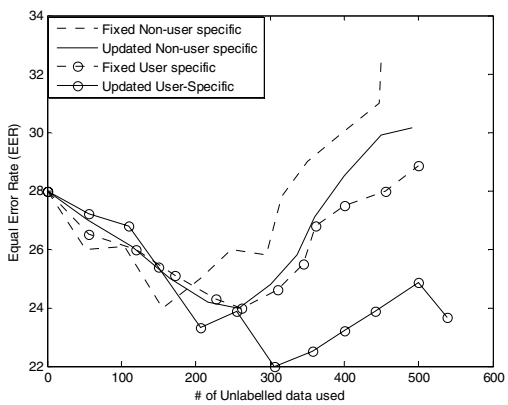

(c)

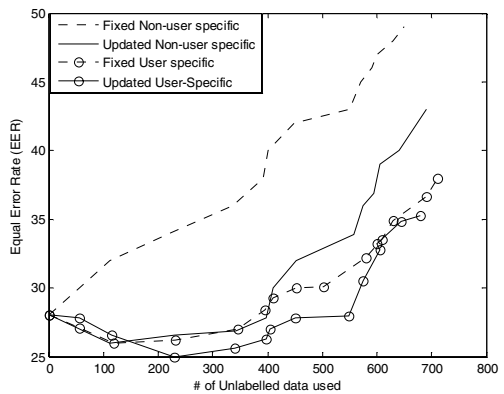

(e)

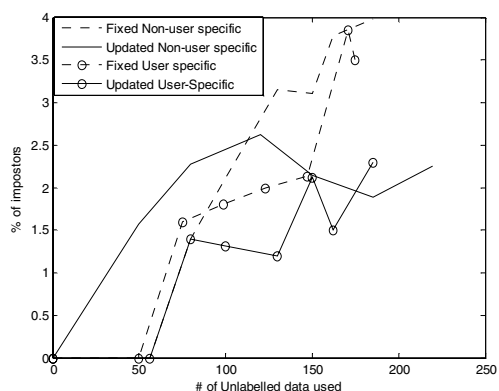

(b)

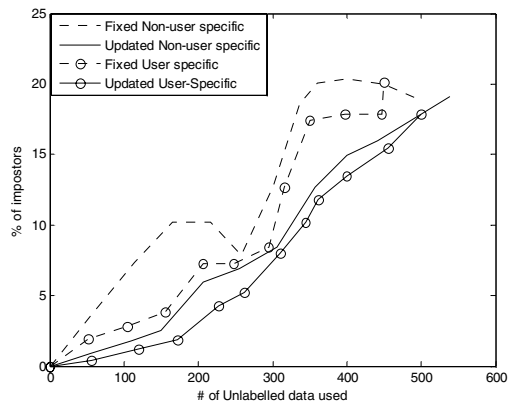

(d)

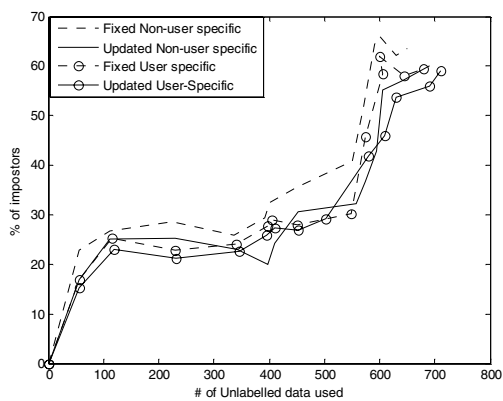

(f)

Fig. 2. Performance obtained in terms of \%EER and \%impostors encountered as a cumulative amount of unlabelled input data added at each iteration by self update for different threshold settings. Plots are reported as follows: (a-b) zeroFAR. (c-d) 1\%FAR. (e-f) 5\%FAR. 


\subsection{The Effect of Wolves and Lambs}

The experimental results reported in Figs. 2(a-f) confirm the accumulative presence of impostors even at the stringent threshold selection conditions. The probabilistic presence of impostors in galleries during automatic self update process is due to basic FAR of the system, which increased very fast as shown in Section 3.3. Although this can be expected at relaxed updating thresholds, but operation at stringent threshold, say zeroFAR, the impostor introduction is still not avoidable. Obviously, a wrong estimation of zeroFAR threshold is to be taken into account. Beside this effect, which causes the introduction of "occasional" impostors, in our opinion the performance degradation can also be due to presence of wolves and lambs, which have so intrinsically overlapping nature (a wolf can be a lamb for others and vice-versa) that an estimation error, even very small, in the threshold at zeroFAR, causes a large number of impostors introduction, despite the low out-degree of the related $\theta$-graph..

Accordingly, we analyzed the updated template galleries at zeroFAR. Some clients were found that acted as wolves, that is, these identities were very frequent among the impostors encountered in the template galleries. According to the Doddington's theory, some wolves were also lambs for other clients as shown by the same person which has been classified as a lamb in the same Table 2. Table 2 shows some impostors identities namely wolves which were always present in most of the updated gallery for all threshold settings. Some corresponding lambs are also reported.. For sake of clarity, values reported in third and fourth columns are related to the case of fixed threshold, as it is the most commonly adopted in literature [2-3]. The third column reports the overall percentage of clients affected by the related wolf, and the

Table 2. Some identities acted as wolves (present in updated templates galleries) for all the threshold selection method (Table 1) at zeroFAR. The current table shows the corresponding lambs, which are always intruded by impostors for all threshold settings (Table 1) at zeroFAR. The statistics related to \%clients affected by the corresponding wolf (column 3) and \% occurrence of samples of the coressponding wolf in updated galleries are presented at fixed-non user specific threshold. Note that at $0 \%$ FAR, $16.5 \%$ identities were not at all updated.

\begin{tabular}{|c|c|c|c|}
\hline \multicolumn{4}{|r|}{$\begin{array}{l}\text { \%samples of } \\
\text { these wolves } \\
\text { in client's } \\
\text { gallery }\end{array}$} \\
\hline & & $18.2 \%$ & $30 \%$ \\
\hline & & $26.67 \%$ & $34 \%$ \\
\hline & & $23.33 \%$ & $19 \%$ \\
\hline & & $16.33 \%$ & $16 \%$ \\
\hline
\end{tabular}


fourth one reports the average percentage of related wolf images among impostors introduced in the gallery of clients they have affected. This motivates why the above clients can be classified as wolves and lambs. The presence of wolves in several clients' galleries pointed out that, even at zeroFAR, the avoidance of intrusions by such identities is crucial for the self update effectiveness.

The effect of wolves can be modelled as in the $\theta$-graph (as discussed in section 2) of Fig. 3. The $\theta$-graph is taken from one of our experiments. For sake of space, it is not complete. The first image on the left-side is the initial template. It can be easily seen again that inclusion of an impostor during self-update process weakens the systems security and make the system vulnerable to others impostors too making the whole process counter-productive. In particular, the initial template of Fig. 3 is in the set of lambs of the fourth wolf of Table 2. It can be noticed that such wolf attracts quickly other samples similar to itself even after few iterations of self update. Finally, another wolf attack can also be seen by the last image (third subject of Table 2).

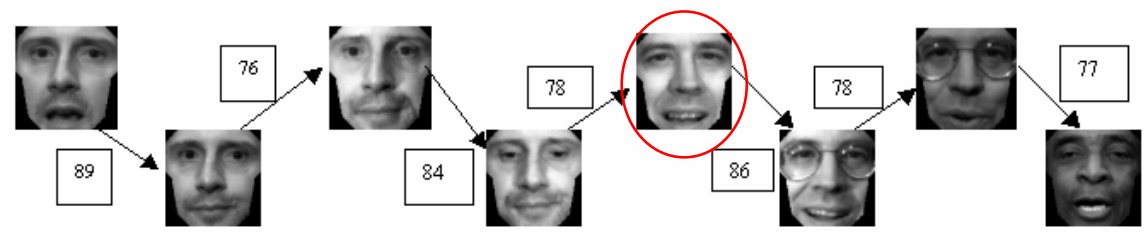

Fig. 3. $\theta$-graph drawn for the selected identity at $0 \%$ FAR at fixed non-user specific threshold. It can be seen that introduction of an impostor makes the system vulnerable to same impostors and other impostors too. Red encircled identity is a wolf (Table 2, last row) which attracts more difficult samples of the same wolf with intra-class variations (spectacles and illumination invariance) and subject the system to other wolf too (last image; Table 2, third row).

Thus, the above experiments highlight that impostor inclusion is a serious issue related to automatic template self-update systems. So far, no method has been proposed to predict or avoid it. The only way to limit impostors introduction, in particular at stringent threshold, where a minimum of EER enhancement has been found, is the appropriate choice of the early stopping criterion of impostors. Since using a cross-validation methodology is not realistic in the case of self update, this criterion to avoid impostors must pass through an appropriate modelling of the impostor's introduction probability. In our opinion, some statistical solutions to predict wolves, lambs, goats, already proposed in the literature, could help to reduce or minimize this difficult task of modelling impostor introduction. For example, in [6] various tests like F-test, Kruskal-Wallis and Durbin tests have been proposed to model these peculiar characteristics of clients. Some criteria to rank the users in a database based on their variability in performance and wolves attack probabilities estimation have been also recently proposed [9-10]. These approaches has not yet been applied to self update algorithms, and may help in designing an appropriate early stopping criterion of impostor's introduction by modelling wolves and lambs probabilities, specially in the case of very stringent updating threshold. However, it is worth remarking that the problem is still an open issue. 


\section{Conclusions}

In this paper, we clearly showed that the self update process is very much dependent on the threshold type settings and the security level for acceptance of input data which have the direct bearing on percentage of impostor introduction and performance degradation. This analysis has not yet been done in the literature on semi-supervised template update methods.

In particular, our investigation was related to self update methods. It has also been found that the impostors inclusion cannot be completely avoided even at strict threshold settings as apart from basic FAR of the system, identities so called wolves and lambs do have adverse effect on the self update process and may be introduced in template galleries of the client. Introduction of impostors has an accumulative adverse effect as they may attract more samples of them and other impostors too, especially if they act as wolves. The operation at optimal threshold value (user specific with updating) together with an appropriate early stopping criterion based on the "wolves and lambs" modelling may lead to reduce or minimize this evident drawback.

\section{References}

1. Uludag, U., Ross, A., Jain, A.: Biometric template selection and update: a case study in fingerprints. Pattern Recognition 37(7), 1533-1542 (2004)

2. Jiang, X., Ser, W.: Online Fingerprint Template Improvement. IEEE Trans. PAMI 24(8), 1121-1126 (2002)

3. Ryu, C., Hakil, K., Jain, A.: Template adaptation based fingerprint verification. In: Proc. of ICPR, Hong Kong, vol. 4, pp. 582-585 (2006)

4. Roli, F., Marcialis, G.L.: Semi-supervised PCA-based face recognition using self-training. In: Yeung, D.-Y., Kwok, J.T., Fred, A., Roli, F., de Ridder, D. (eds.) SSPR 2006 and SPR 2006. LNCS, vol. 4109, pp. 560-568. Springer, Heidelberg (2006)

5. Rattani, A., Marcialis, G.L., Roli, F.: Capturing large intra-class variations of biometric data by template co-updating. In: IEEE Workshop on Biometrics, Int. Conf. on Vision and Pattern Recognition CVPR 2008, Anchorage, Alaska, USA (in press, 2008)

6. Doddington, G., Liggett, W., Martin, A., Przybocki, M., Reynolds, D.: Sheeps, Goats, Lambs and Wolves: A Statistical Analysis of Speaker Performance in the NIST 1998 Speaker Recognition Evaluation. In: Proc. ICSLD, Sydney, Australia (1998)

7. Wiskott, L., Fellows, J.M., Kruger, N., von der Malsburg, C.: Face recognition by elastic bunch graph matching, Technical Report IR-INI, 96-08 (1996)

8. Equinox Face Database, http://www.equinoxsensors.com/products/HID.html

9. Poh, N., Bengio, S., Ross, A.: Revisiting Doddington's Zoo: A Systematic Method to Assess User-dependent Variabilities. In: Proc. of 2nd Work. on Multimodal User Authentication (MMUA), Toulose, France (May 2006)

10. Une, M., Otsuka, A., Imai, H.: Wolf Attack Probability: A New Security Measure in Biometric Authentication Systems. In: Lee, S.-W., Li, S.Z. (eds.) ICB 2007. LNCS, vol. 4642, pp. 396-406. Springer, Heidelberg (2007) 